\title{
Electron beam requirements for a three-dimensional Smith-Purcell backward-wave oscillator for intense terahertz radiation
}

\author{
Kwang-Je Kim ${ }^{1}$ and Vinit Kumar ${ }^{2}$ \\ ${ }^{1}$ Argonne National Laboratory, 9700 S. Cass Avenue, Argonne, Illinois 60439, USA \\ ${ }^{2}$ Raja Ramanna Center for Advanced Technology, Indore, India
}

(Received 7 June 2006; published 22 August 2007)

\begin{abstract}
A Smith-Purcell device can operate as a backward-wave oscillator for intense, narrow-bandwidth, continuous wave radiation at terahertz wavelengths. We determine the requirements on electron beam current and emittance for the system to oscillate based on a three-dimensional extension of our previous two-dimensional analysis. It is found that specially designed electron beams are required with a current that exceeds a certain threshold value and a flat transverse profile that allows the beam to travel very close to the grating surface. Two methods for producing electron beams with the required characteristics are discussed.
\end{abstract}

DOI: 10.1103/PhysRevSTAB.10.080702

\section{INTRODUCTION}

The interest in a Smith-Purcell device, first proposed and experimentally tested over half a century ago [1], was rekindled recently when a Dartmouth group constructed a tabletop terahertz source by retrofitting the sample chamber of a scanning electron microscope (SEM) with a metal grating [2]. The radiation intensity after a certain threshold was reported to increase as a fourth power in the electron beam current. The behavior was interpreted as arising from a gain process similar to that occurring in a free-electron laser (FEL). If confirmed, the device, due to its compact size and high-intensity output, will have a great impact on terahertz science, which suffers from a scarcity of readily available sources [3].

An effort to reproduce the Dartmouth result at the Enrico Fermi Institute (EFI) in the University of Chicago with a Smith-Purcell device closely resembling the Dartmouth design [4] has not been successful. Although prominent signals exhibiting nonlinear behavior were indeed observed in the initial measurements, they were reduced drastically to near noise level when a cooling channel was introduced to the copper block attached to the grating. Therefore, the initial signal was likely due to the blackbody radiation from the heated grating surface onto which the electron beam was swept periodically.

While the EFI experiment was not successful, we should still expect that a Smith-Purcell device can be made to "lase" if certain conditions on electron beam qualities are met. It is therefore important to ask what those conditions are and whether it is feasible to meet those conditions. These are the questions we address in this paper.

The gain in a Smith-Purcell radiator occurs when electron beam interacts with a copropagating surface mode, which is an evanescent electromagnetic mode near the grating surface [5]. A consistent linear analysis of the electron beam interacting with the surface mode was presented by Andrews and Brau [6]. They also made a crucial
PACS numbers: 52.59.-f, 41.60.Cr, 41.20.-q, 41.85.Ew

observation that the surface mode is a backward wave, i.e., its group velocity is in the direction opposite to its phase velocity. In this case the device can operate as an oscillator, even in the absence of external mirrors, due to the distributed feedback arising from backward propagation of the optical energy. Such a device is known as the backwardwave oscillator (BWO) in the microwave literature [7].

The results in Ref. [6] are not directly useful in deriving the criteria for the characteristics of the electron beam since the analysis was for the case of an electron beam filling uniformly the entire half space above the grating. However, we have recently developed a nonlinear theory for a two-dimensional Smith-Purcell BWO in which the electron distribution is infinitely wide and uniform in the groove direction and has an arbitrary profile in the direction normal to the grating surface [8]. The special case of this theory with a thin electron beam is a good starting point for studying a practical Smith-Purcell BWO for terahertz generation since the required beam profile turns out to be very thin.

When the surface mode is a forward wave, the gain process is similar as in a traveling wave amplifier (TWA). Devices based on a Smith-Purcell TWA have been studied for two-dimensional configurations by several authors in the past, including the Smith-Purcell oscillator by Schachter and Ron [9] and an amplifier by Yasumoto et al. [10]. Lacking a natural feedback mechanism, however, the TWA devices require either an optical cavity with a high round trip reflectivity or a very high single-pass gain. Therefore, we concentrate on the BWO devices in this paper.

A three-dimensional theory is necessary to discuss parameters for a realistic electron beam with a finite width that can drive a Smith-Purcell BWO. Fortunately, it turns out that the three-dimensional extension of the twodimensional theory presented in Ref. [8] is simple and straightforward. We are therefore able to determine the requirements of electron beam properties to operate a 
practical Smith-Purcell BWO for terahertz generation; we found that the electron beam current must exceed a certain threshold value and the profile of the electron beam should be thin and flat so that the beam can be placed close to the grating surface. The experiments at Dartmouth and at EFI did not satisfy these requirements. We discuss two possible approaches to produce such electron beams, one by a line source and one by a phase-space manipulation of an initially round beam. Using these beams, a Smith-Purcell BWO capable of producing a few watts of terahertz power can be constructed.

In Sec. II, we review the two-dimensional theory of interaction between an electron beam of vanishing thickness and infinite width and a surface mode of a grating [8]. Extension of the theory to the case of finite beam width, and paraxial mode propagation is discussed in Sec. III. We obtain in Sec. IV the requirements on electron beam characteristics for operation of a BWO. We then discuss an explicit example for a THz source in Sec. V. Section VI is devoted to a discussion of two methods for production of a suitable quality electron beam for such a device. Finally, we present some conclusions in Sec. VII.

\section{SMITH-PURCELL BWO: TWO-DIMENSIONAL THIN BEAM CASE}

The coordinate system we use in this paper is shown in Fig. 1 and is as follows: the $x$-direction is along the grating groove, the $y$-direction is normal to the grating surface, and the $z$-direction is on the grating surface but perpendicular to the groove direction. The grating extends uniformly to the positive and negative $x$-direction. The length of the grating and its period are $L$ and $\lambda_{g}$, respectively. There are three plane segments in each grating period; the bottom segments of width $w$ at $y=-h$, the top segments at $y=0$, and segments connecting the top and bottom segments.
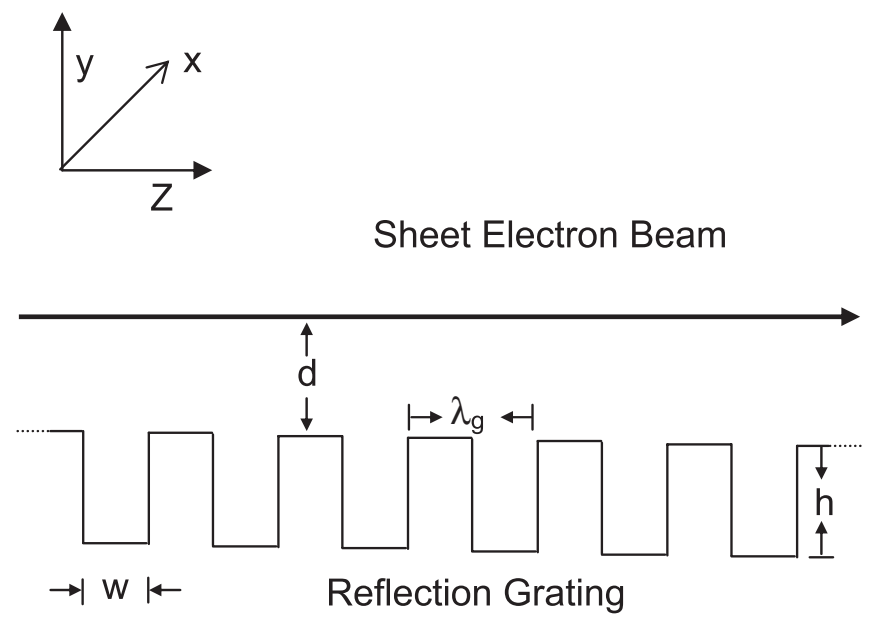

FIG. 1. The grating profile and the coordinate system. The $x$-direction is into the page.
A surface mode is a solution of Maxwell equation satisfying suitable boundary conditions in the absence of a driving electron current. We assume harmonic time dependence $\exp (-i \omega t)$. The grating may be regarded as a wave guide in the $x$-direction, and we consider the modes for which the $x$-component of the electric field $E_{x}$ vanishes and other field components are determined from the $x$-component of the magnetic field $B_{x}$. (Although the $z$-component of the magnetic field vanishes, these are properly the TE modes in the waveguide terminology since the axis of the waveguide in the present case is in the $x$-direction.) In this section we consider the twodimensional situation in which all quantities are independent of the $x$-coordinate. Taking into account the grating periodicity, the field can be written as a sum of space harmonics. Thus we write the $z$-component of the electric field as follows:

$$
E_{z}=\sum_{n} A_{n} e^{i\left(k_{z}+n k_{g}\right) z} e^{-\Gamma_{n} y},
$$

where $k_{z}$ is the propagation wave number for the $n=0$ term in the $z$-direction, $k_{g}=2 \pi / \lambda_{g}, k=\omega / c=2 \pi / \lambda, c$ is the speed of light, $\lambda$ is the free space wavelength, and

$$
\Gamma_{n}=\sqrt{\left(k_{z}+n k_{g}\right)^{2}-k^{2}}
$$

The boundary condition on the grating surface determines the relative magnitudes of the amplitudes $A_{n}$ as well as the relation between $k_{z}$ and $k$, i.e., the dispersion relation as discussed in Refs. [8,11].

Consider now an electron beam, infinitely thin in the $y$-direction, propagating in the $z$-direction at a distance $y=$ $d$ above the grating. A sustained interaction can occur between the surface mode and electron beam if the phase velocity of the $n=0$ term is the same as the electron velocity:

$$
\frac{k}{k_{z}}=\beta .
$$

Here $\beta$ is the electron velocity in units of the speed of light. The group velocity on the other hand is given by $d \omega / d k_{z}$, which can be computed from the dispersion relation of the surface mode. For cases of interest in this paper, the group velocity of the copropagating surface mode turns out to be backward, i.e., along the negative $z$-axis [6]. In the following, the magnitude of the group velocity will be denoted by $v_{g}$. The $n=0$ term of the copropagating $E_{z}$ field at the location of the electron beam $y=d$ is written as $E(z, t) \times$ $\exp \left(i k_{z} z-i \omega t\right)$, where $E(z, t)$ is the amplitude, which varies slowly due to interaction with the electron beam. The equation for the evolution of the amplitude $E(z, t)$ derived earlier [8] and generalized to include the wave attenuation [12] is as follows:

$$
\frac{\partial E}{\partial z}-\frac{1}{v_{g}} \frac{\partial E}{\partial t}=\frac{I Z_{0} \chi}{4 \beta \gamma \Delta x} e^{-2 \Gamma_{0} d}\left\langle e^{-i \theta}\right\rangle+\alpha E,
$$


where the first term on the left-hand side of this equation describes transport of the optical energy to the backward direction, i.e., along the negative $z$-direction. The righthand side represents the electron-mode coupling, in which $I$ is the electron beam current, $\Delta x$ is the half width of the beam in the $x$-direction assumed to be large so that the twodimensional approximation is valid, $\gamma$ is the electron energy in units of rest energy, $Z_{0}=377 \Omega$ is the characteristic impedance of free space, $\Gamma_{0}=k / \beta \gamma, \theta$ is the electron phase, and $\chi$ is the residue of the singularity associated with the surface mode as defined in Ref. [8]. The factor $e^{-2 \Gamma_{0} d}$ in Eq. (4), which will be important for our discussion of the desirable electron beam profile, arises from the fact that the force acting on the beam is mediated by the grating surface. The second term on the right-hand side of Eq. (4) represents attenuation due to the finite conductivity of the grating material, where the attenuation coefficient $\alpha$ can be obtained by the prescription given by Andrews et al. [13].

The dynamics of the system is determined by the Maxwell equation given by Eq. (4) together with the Lorentz equation for the electron motion under the influence of the surface mode. If the mode is a forward wave, as in the usual FEL, the mode amplitude will be amplified as it propagates together with the electron beam in the positive $z$-direction. Oscillation occurs in this case only if the amplified radiation is fed back to the entrance of the interaction region by placing the system within an optical cavity. In the case of a backward wave considered in this paper, however, the oscillation is possible even without feedback mirrors [7]. Indeed, by linearizing the equations and solving them with the boundary condition appropriate for the backward-wave case, $E(z=L, t)=0$ for all $t$, we find that the system may exhibit an absolute instability, i.e., the mode amplitude grows exponentially at all points in the interaction region $0 \leq z<L$ [14]. The condition for this instability is that the linear density of the current $d I / d x$ exceeds a threshold value $d I_{S} / d x$ :

$$
\frac{d I}{d x}>\frac{d I_{S}}{d x}=\mathcal{J}_{S}(\eta) \frac{I_{A}}{2 \pi \chi} \frac{\beta^{4} \gamma^{4}}{k L^{3}} e^{2 \Gamma_{0} d}
$$

Here $\mathcal{J}_{S}(\eta)$ is the dimensionless start current as a function of the loss parameter $\eta=\alpha L$ and $I_{A}=17 \mathrm{kA}$ is the Alfvén current. Equation (5) generalizes the corresponding inequality for the special case of a perfect conductor $\eta=0$ [8]. The quantity $d I_{S} / d x$ will be referred to as the linear density of the start current.

The exponential growth will stop eventually and the system will reach a steady state. The behavior near the "saturation" is nonlinear and can only be determined accurately by numerically solving the Maxwell-Lorentz equation, as was done in Ref. [8], or by using the PIC code, as was done in Refs. [15-19].

\section{LOCALIZATION OF SURFACE MODE IN THE HORIZONTAL DIRECTION}

For practical application, the two-dimensional thin beam theory we reviewed in the previous section needs to be extended for the three-dimensional case where the surface mode has a finite extent in the $x$-direction. Since the grating is open in the $x$-direction, we expect that the surface mode will undergo diffraction in the $(x, z)$ plane similar to freely propagating optical beam. We can construct a surface mode in such a case by combining the plane waves propagating at different angles in the $(x, z)$ plane as follows:

$$
E_{z}=\sum_{n} \int d k_{x} A_{n}\left(k_{x}\right) e^{i\left(k_{z}+n k_{g}\right) z} e^{i k_{x} x} e^{-\Gamma_{n} y} .
$$

The analysis in the last section is a special case with $k_{x}=$ 0 . It is now necessary to find a modified dispersion relation for nonvanishing $k_{x}$. By retracing the derivation in Ref. [9], we find the simple result that the quantities $k$ and $k_{x}$ occur only through the quantity $\sqrt{k^{2}-k_{x}^{2}}$ in the impedance matrix defined there. Leaving the details of the derivation to another publication [20], we can therefore state the following simple result: For a given $k$, the value of $k_{z}$ for a nonvanishing $k_{x}$ is the same as the value of $k_{z}$ obtained for the $k_{x}=0$ case provided we replace $k$ with $\sqrt{k^{2}-k_{x}^{2}}$.

It is now convenient to introduce the angle via $k_{x}=$ $k_{z} \sin \phi$. Under the paraxial approximation $\phi \ll 1$, the quantities involving $\phi$ may be expanded in a Taylor series, and we find the following expressions for the $\varphi$-dependence of $k_{z}$ and $\Gamma_{0}$ :

$$
\begin{aligned}
k_{z}(\phi) & =k_{z}\left(1+\frac{\phi^{2}}{2 \beta \beta_{g}}\right), \\
\Gamma_{0}(\phi) & =\Gamma_{0}+\frac{k_{z}^{2} \phi^{2}}{2 \Gamma_{0}}\left(1+\frac{1}{\beta \beta_{g}}\right),
\end{aligned}
$$

where we simplified the notation by writing $k_{z}=k_{z}(0)$ and $\Gamma_{0}=\Gamma_{0}(0)$. Once we have derived the expression for the $n=0$ term of the surface mode propagating at an angle in the $(x, z)$ plane, we combine these in the following manner with a suitable weight function $A_{0}(\phi)$ to obtain a mode localized in $(x, z)$ plane

$$
\begin{aligned}
E_{z}(x, y, z)= & \int A_{0}(\phi) e^{i\left[\left(k_{z} \phi^{2} z\right) /\left(2 \beta \beta_{g}\right)\right]} \\
& \times e^{-\left[\left(k_{z}^{2} \phi^{2}\right) /\left(2 \Gamma_{0}\right)\right]\left[1+\left[1 /\left(\beta \beta_{g}\right)\right]\right\} y} e^{-\Gamma_{0} y} e^{i k_{z} \phi x} \\
& \times e^{i k_{z} z} d \phi .
\end{aligned}
$$

The rms size of the surface mode in the $x$-direction may be introduced as follows:

$$
\Sigma_{x}^{2}(y ; z)=\frac{\int x^{2}\left|E_{z}(x, y, z)\right|^{2} d x}{\int\left|E_{z}(x, y, z)\right|^{2} d x} .
$$

The integration can be performed if we choose $A_{0}(\phi)=$ $\exp \left(-\phi^{2} / 4 \sigma_{\phi}^{2}\right)$ with the result: 


$$
\Sigma_{x}^{2}(y ; z)=\Sigma_{x}^{2}(y ; 0)+\Sigma_{\phi}^{2}(y) z^{2} .
$$

The equation is in the form of paraxial diffraction with a waist at $z=0$, the rms waist beam size of $\Sigma_{x}(y ; 0)$, and an rms diffraction angular divergence of $\Sigma_{\phi}(y)$. The quantities on the right-hand side of Eq. (10) are determined by the following relations:

$$
\begin{gathered}
\Sigma_{x}^{2}(y ; 0)=\sigma_{x}^{2}+\frac{y}{2 \Gamma_{0}}\left(1+\frac{1}{\beta \beta_{g}}\right), \\
\sigma_{x} \sigma_{\phi}=\frac{1}{2 k_{z}}, \\
\Sigma_{x}(y ; 0) \Sigma_{\phi}(y)=\frac{1}{2 k_{z} \beta \beta_{g}}=\frac{\lambda}{4 \pi \beta_{g}} .
\end{gathered}
$$

These reduce to the well-known relations between the rms size and angular divergence in free space when $\beta_{g}=1$. Equation (10) can also be written in a form familiar in paraxial optics discussions:

$$
\Sigma_{x}^{2}(y ; z)=\frac{\lambda}{4 \pi \beta_{g}}\left(Z_{R}+\frac{z^{2}}{Z_{R}}\right),
$$

where the Rayleigh range $Z_{R}$ is given by

$$
Z_{R}=\frac{4 \pi \beta_{g}}{\lambda} \Sigma_{x}^{2}(y ; 0)
$$

Paraxial diffraction of a wave has a close parallel with the propagation of a particle beam of a finite emittance [21]. In the present case, the quantities $\lambda / 4 \pi \beta_{g}$ and $Z_{R}$ play the role of emittance and beta function, respectively, in particle beam optics.

In the next section, these results will be used to make an estimate for the effect of three-dimensional effects on the performance of Smith-Purcell (SP)-BWOs. There, we will find that the second term in Eq. (11) proportional to $y$ is negligible and can be dropped. Hence the beam size, angular divergence, and the Rayleigh range can all be regarded as independent of the $y$-coordinate.

\section{ELECTRON BEAM REQUIREMENTS}

Before discussing the transverse profile of the electron beam desirable for operation of a Smith-Purcell BWO, it is useful to review the beam properties in phase space. For clarity, we will assume that the electron beam distribution in the four-dimensional phase space $(x, \phi, y, \psi)$ is given by the Kapchinskij-Vladimirskij (KV) distribution [22], where $x$ and $y$ are the horizontal and vertical coordinates, respectively, and $\phi$ and $\psi$ are the horizontal and vertical angles, respectively. The KV distribution is convenient because the distribution in any two-dimensional subspace is uniform within an ellipse. We consider the distribution in the middle of the grating and assume that the twodimensional beam ellipses are all upright with the half widths $(\Delta x, \Delta \phi, \Delta y, \Delta \psi)$. The half widths are related to the rms values by a factor of 2 ; $(\Delta x, \Delta \phi, \Delta y, \Delta \psi)=$ $2\left(\sigma_{x}, \sigma_{\phi}, \sigma_{y}, \sigma_{\psi}\right)$. The product $\varepsilon_{x}{ }^{0}=\sigma_{x} \sigma_{\phi}=$ $(1 / 4) \Delta x \Delta \phi$ is known as the geometric rms emittance in the $x$-direction and is a measure of the beam quality. The normalized $\mathrm{rms}$ emittance is defined by $\varepsilon_{x}=\beta \gamma \varepsilon_{x}{ }^{0}$, which is invariant under acceleration. The quantity $\beta_{x}=$ $\sigma_{x} / \sigma_{\phi}=\Delta x / \Delta \phi$ is known as the Courant-Snyder envelope (in the middle of the grating) in the $x$-direction and is determined by the beam transport design. Similar quantities are defined in the $y$-direction with the subscript $y$. The emittance can also be written as $\varepsilon_{x}{ }^{0}=\sigma_{x}^{2} / \beta_{x}$. The rms $x$-beam size at the end of the grating is given by $\sqrt{\varepsilon_{x}{ }^{0} \beta_{x}\left[1+\left(L / 2 \beta_{x}\right)^{2}\right]}$. We need to choose $\beta_{x} \geq L$ and similarly in the $y$-direction so that the beam sizes do not vary much along the length of the grating. For a given beam size, we need to choose $\beta_{x}=L$ to maximize the emittance, keeping the beam size more or less constant along the length of the grating.

The optimum beam profile corresponds to the minimum of the linear density of the start current. In view of the last factor in Eq. (5), $e^{2 \Gamma_{0} d}$, we should require that the electrons' vertical separation from the grating surface satisfy $d \leq 1 / 2 \Gamma_{0}$. Assuming that the beam edge just touches the grating surface, we require $\Delta y=1 / 2 \Gamma_{0}$. Choosing the envelope $\beta_{y}=L$ to avoid making the emittance requirement too stringent, we obtain

$$
\varepsilon_{y} \leq \frac{\beta \gamma}{\left(4 \Gamma_{0}\right)^{2}} \frac{1}{L} .
$$

This condition turns out to be very stringent.

Let us now turn to the requirement in the $x$-direction. From the analysis presented in the last section, we find that the geometric rms emittance, i.e., the product of the rms beam size and the divergence for the copropagating evanescent wave, is $\lambda / 4 \pi \beta_{g}$. Hence, the normalized rms emittance of the electron beam in the $x$-direction should satisfy

$$
\varepsilon_{x} \leq \beta \gamma \frac{\lambda}{4 \pi \beta_{g}} .
$$

The Rayleigh range $Z_{R}$ for an optical wave packet is the quantity corresponding to the envelope for an electron beam. To avoid large variation in the optical beam size, we will set $Z_{R}=L$. The choice may require the use of external mirrors of suitable curvature. In the situation where it is not possible to control the Rayleigh range, it will be decided by the boundary conditions at the ends of the grating. It will be complicated to obtain $Z_{R}$ for the most general case. Here, we assume that it will be possible to set $Z_{R}=L$. In a real situation, if $Z_{R}$ has a different value, then one will have to change the value of $Z_{R}$ in the analysis presented here. The electron beam envelope should be suitably chosen so that the electron beam size is the same 
as the optical beam size to maximize the overlap:

$$
\Delta x=2 \sqrt{\frac{\lambda L}{4 \pi \beta_{g}}} .
$$

If the electron beam emittance in the $x$-direction is significantly less than the right-hand side of Eq. (17), Eq (18) can still be satisfied by choosing $\beta_{x} \gg L$.

In a three-dimensional situation, we may interpret the linear density of the start current $d I_{S} / d x$ as the peak value in the middle of the electron beam distribution. The threshold condition in Eq. (5) then becomes a condition on total current $I$ :

$$
I>I_{S}=\frac{\pi}{2} \frac{d I_{S}}{d x} \Delta x
$$

The inequalities of Eqs. (16), (17), and (19) are the basic requirements for operation of a Smith-Purcell BWO. If these conditions are satisfied, the intensity of the surface mode in the grating region rises exponentially until it reaches saturation, and from then on the device operates in a steady state. The efficiency at saturation - the ratio of the optical energy generated to the electron beam's kinetic energy - can be estimated from the amount of electron energy loss corresponding to its slipping behind by a distance of $\lambda_{z} / 4$ [8]. For the nonrelativistic case, the efficiency is given by $\eta_{\text {eff }} \approx \lambda_{z} / 2 L$. We emphasize that this is only an order of magnitude estimate since it is based on simple considerations and many realistic effects like attenuation due finite conductivity of grating material, the reflection at the ends of the grating, etc., are not taken into account while deriving this formula. The optical power appears partly in the form of diffracted wave at the grating entrance and partly in the form of propagating waves at higher harmonics due to the higher harmonic components of the density modulation in the electron beam [23]. Since the electron beam power at threshold is $P_{S}^{\text {ebeam }}=V I_{S}$, where $V$ is the beam voltage, the optical output power is therefore $P^{\mathrm{opt}} \geq P_{S}^{\mathrm{opt}}=\eta_{\mathrm{eff}} V I_{S}$.

\section{AN EXAMPLE CASE}

We now wish to discuss an explicit example. The electron's kinetic energy $q V$ ( $q=$ electron charge) is taken to be $35 \mathrm{keV}$, corresponding to $\beta=0.352(\beta \gamma=0.376)$. For grating, we set the period $\lambda_{g}=173 \mu \mathrm{m}$ and the total length $L=19.03 \mathrm{~mm}$. These are the same as in the Dartmouth experiment [2] except that the grating length is increased by $50 \%$ in order to reduce the start current. For the grating profile, we took the parameters for the Dartmouth experiment, i.e., the width of the bottom segment $w=62 \mu \mathrm{m}$ and the height of the top segment $h=$ $100 \mu \mathrm{m}$ as the base parameter and then further optimized them for the minimum start current. To compute the attenuation coefficient $\alpha$ for copper grating, we have followed the procedure described in Ref. [13] using the DC conductivity $\sigma_{0}=5.76 \times 10^{7} / \Omega$-m and the electron relaxation time $\tau=2.4 \times 10^{-14} \mathrm{~s}$. The dimensionless start current $\mathcal{J}_{S}(\alpha L)$ is then obtained by solving a cubic equation as discussed in Ref. [12]. The resonant wavelength $\lambda$, the group velocity $v_{g}$, and the parameter $\chi$ can be obtained using the procedure discussed in Ref. [8]. The start current $I_{S}$ can then be obtained by substituting these parameters in Eqs. (5), (18), and (19). The result as a function of $h$ is plotted as a solid curve in Fig. 2, which shows that it decreases initially as $h$ is increased from $100 \mu \mathrm{m}$, reaching a minimum $I_{S}=46 \mathrm{~mA}$ at around $h=130 \mu \mathrm{m}$, and increases thereafter. We therefore choose $h=130 \mu \mathrm{m}$. Then the procedure is repeated for optimizing the groove width $w$ to minimize $I_{s}$, keeping $h=130 \mu \mathrm{m}$. This is shown by the dashed curve in Fig. 2 where the optimum groove width $w$ is seen to be $110 \mu \mathrm{m}$, for which $I_{s}=$ $37.2 \mathrm{~mA}$. As discussed earlier $2 \Gamma_{0} d=1$ in the calculation here. The free-space wavelength of the surface mode for these optimized parameters is then found to be $\lambda=$ $761 \mu \mathrm{m}$, the group velocity $v_{g}=0.184 c, \quad \Gamma_{0}=$ $0.0222 \mu \mathrm{m}^{-1}, \Delta y=22.6 \mu \mathrm{m}$, and the required emittance in the $y$-direction from Eq. (16) is very small: $\varepsilon_{y} \leq 2.5 \times$ $10^{-9} \mathrm{~m}$-r. The emittance in the $x$-direction from Eq. (17) can be much larger: $\varepsilon_{x} \leq 120 \times 10^{-6} \mathrm{~m}$-r. The half width of the beam in the $x$-direction from Eq. (18) is $\Delta x=$ $5.00 \mathrm{~mm}$.

A simulation of Smith-Purcell BWO was performed by solving the Maxwell-Lorentz equations [8,12]. The energy spread of the electron beam is not included in this calculation since the spread produced by electron guns under consideration is negligible. The three-dimensional effects were included in the approximate manner as discussed.

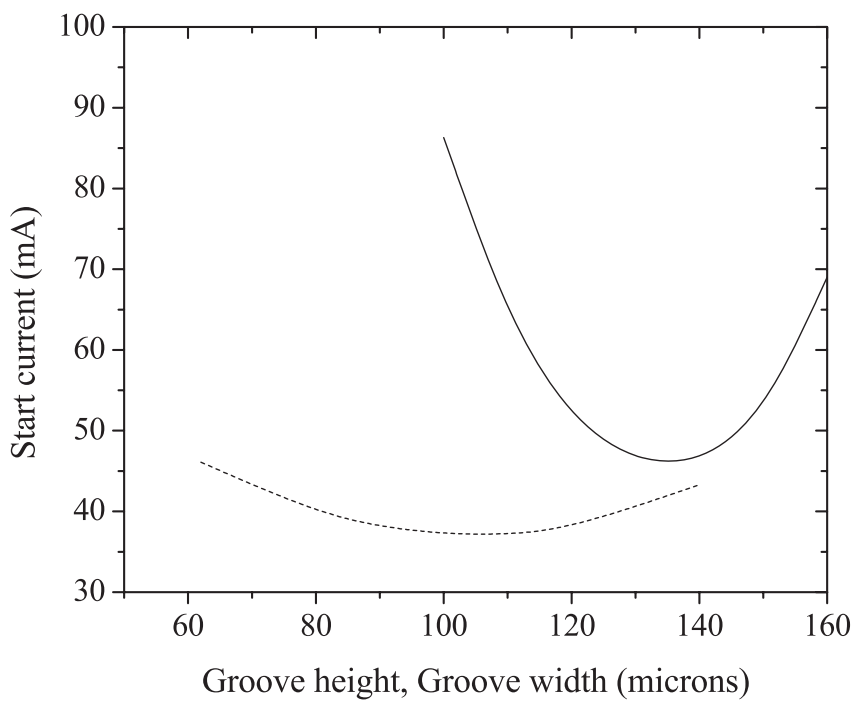

FIG. 2. Start current as a function of the groove height $h$ (solid curve) as well as groove width (dashed curve) for grating and electron beam parameters as discussed in the text. For the solid curve, we have kept the groove width fixed at $62 \mu \mathrm{m}$. For the dashed curve, the groove height is fixed at $130 \mu \mathrm{m}$. 
More elaborate PIC simulations have been performed recently by several authors [15-19]. Compared to the results of three-dimensional PIC simulations by Li et al. [16], our simulation for the parameters mentioned there gives the amplitude of saturated longitudinal electric field, which is around $17 \%$ larger compared to the that obtained using PIC code [24]. Compared to the results of two-dimensional PIC simulation reported by $\mathrm{Li}$ et al. [17], the start current obtained by us using Eq. (5) is only 13\% lower [24]. Finally, compared to the results of two-dimensional simulation by Donohue et al. [18], our result for the amplitude of saturated magnetic field for the parameters mentioned in Ref. [18] is in excellent agreement [24]. We note that our simulation based on Maxwell-Lorentz equations is much faster compared to the PIC codes. A detailed description of the comparison of our simulation with more elaborate PIC simulation can be found in Ref. [23].

For the case we discussed in the previous paragraph, Fig. 3 shows the evolution of power in the surface mode for a beam current of $45 \mathrm{~mA}$. Note that we have plotted the total power in the surface mode, which includes electromagnetic field in all space harmonics in Eq. (1). We have discussed the calculation of power in detail earlier in Ref. [8]. It can be seen in Fig. 3 that the saturated power is $3.9 \mathrm{~W}$, which corresponds to efficiency $\eta_{\text {eff }} \approx 0.3 \%$ since the power in the electron beam for these parameters is $P_{\text {ebeam }}=1.35 \mathrm{~kW}$. Using the approximate formula for the efficiency derived in the previous paragraph, the estimated efficiency $\eta_{\text {eff }} \approx 0.7 \%$. The reduced efficiency in the simulation is due to attenuation and also due to nonlinear effects; the efficiency is improved at higher current. Hence, we conclude that this system can generate a continuous wave terahertz output of the order of a few Watts, an intensity level that has not been available with a compact, laboratory-scale device.

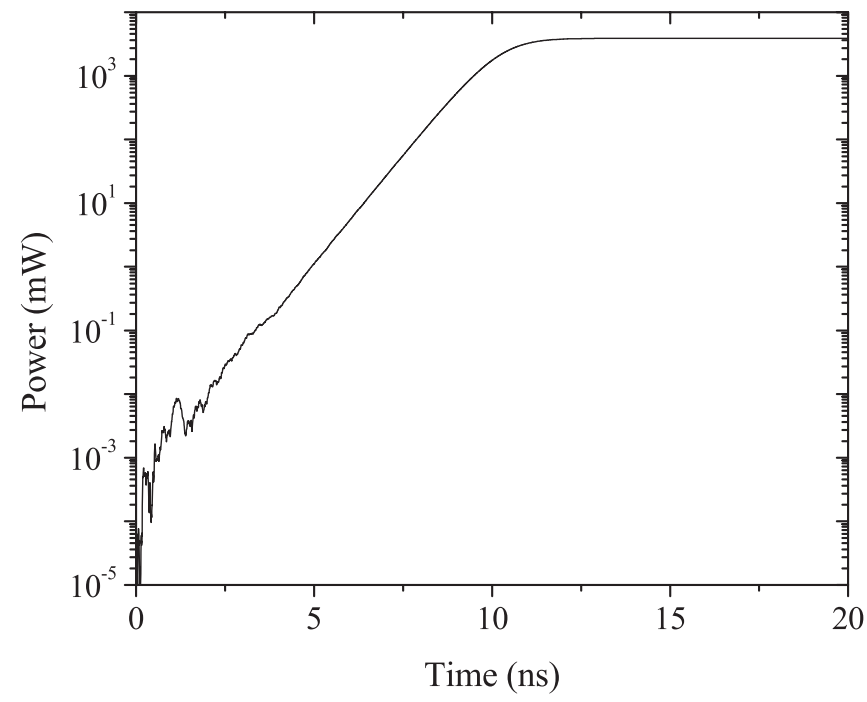

FIG. 3. Plot of power as a function of time in the surface mode at the entrance of the grating for the optimized parameters discussed in the text. We have taken $I=45 \mathrm{~mA}$.

\section{BEAM PRODUCTION}

Can such an electron beam to create coherent $\mathrm{THz}$ radiation be produced? For an elliptically shaped, planar, thermionic cathode with the major (minor) axis of $\Delta x_{c}$ $\left(\Delta y_{c}\right)$, the thermal emittances in the $x$ - and $y$-directions are given by $\left(\varepsilon_{c x}, \varepsilon_{c y}\right)=0.5\left(\Delta x_{c}, \Delta y_{c}\right) \sqrt{k_{B} T / m c^{2}}$, where $k_{B}$ is Boltzmann's constant, $T$ is the absolute temperature, and $m$ is the electron mass. For $T=2500 \mathrm{~K}$, the square root factor is $0.65 \times 10^{-3}$. The cathode dimensions satisfying the emittance requirements discussed in the previous section are $\Delta x_{c} \leq 37 \mathrm{~cm}$ and $\Delta y_{c} \leq 8 \mu \mathrm{m}$. The cathode is therefore a long, thin line source. The current density at the cathode, corresponding to the current of $45 \mathrm{~mA}$, is $j_{c}=$ $I_{S} / \pi \Delta x_{c} \Delta y_{c}=0.5 \mathrm{~A} / \mathrm{cm}^{2}$, well within the range of an off-the-shelf tungsten cathode for SEMs producing $j_{c}=$ 1-3 A/ $\mathrm{cm}^{2}$ [25].

We need to check that the space-charge effect does not blow up the emittance. This will be the case if the emittance term is smaller than the space-charge term in the beam envelope equation [22]. The condition in the $y$-direction can be written as

$$
\frac{1}{4 \beta \gamma} \frac{I}{I_{A}} \frac{\Delta y^{3}}{(\Delta x+\Delta y) \varepsilon_{y}{ }^{2}}<1
$$

If the left-hand side is evaluated with the beam parameters at $45 \mathrm{~mA}$ and $35 \mathrm{keV}$, we obtain 0.7 , thus the inequality is satisfied. The condition in the $x$-direction is less restrictive. The magnitude of the emittance dilution while the beam is accelerated depends on the acceleration gradient and can only be determined by detailed simulation.

Another possibility is the novel phase-space technique, in which a round electron beam is produced from a cathode immersed in an axial magnetic field, and then the beam angular momentum is removed by a set of quadrupoles $[26,27]$. The technique was recently demonstrated experimentally $[28,29]$. In this scheme, the ratio of the final emittances is given by

$$
\frac{\varepsilon_{x}}{\varepsilon_{y}}=\left(\frac{q B}{m c} \frac{r_{c}^{2}}{4 \varepsilon_{I}}\right)^{2}
$$

where $B$ is the magnetic field at the cathode; $r_{c}$ is the cathode radius; and $\varepsilon_{I}=\sqrt{\varepsilon_{x}} \overline{\varepsilon_{y}}$ is the initial, roundbeam emittance before emission into the axial magnetic field. Using $r_{c}=2 \varepsilon_{I} / \sqrt{k_{B} T / m c^{2}}$, we find that the required magnetic field can be written as $B=\left(k_{B} T\right) /\left(q \varepsilon_{y} c\right)$. Note that this is independent of $\varepsilon_{x}$. Choosing the maximum allowed value $\varepsilon_{y}=2.5 \times 10^{-9} \mathrm{~m}-\mathrm{r}$ and assuming a thermionic cathode with $T=2500 \mathrm{~K}$ as in the previous paragraph, we obtain $B=2.9 \mathrm{kG}$, a modest value that can be generated either by a permanent magnet or an electromagnet. Let us take $\varepsilon_{x}=2.5 \times 10^{-6} \mathrm{~m}-\mathrm{r}$, smaller by a factor of 48 than the maximum allowed value, $120 \times$ $10^{-6} \mathrm{~m}$-r. The emittance ratio $\varepsilon_{x} / \varepsilon_{y} \approx 1000$ appears to 
be feasible, although it is approximately an order of magnitude larger than the ratio achieved recently [29]. The round-beam emittance is $\varepsilon_{I} \approx 7.9 \times 10^{-8} \mathrm{~m}-\mathrm{r}$, and the corresponding radius is $r_{c}=243 \mu \mathrm{m}$. The current density at threshold is $j_{c}=24 \mathrm{~A} / \mathrm{cm}^{2}$, which should be feasible with a tungsten cathode. The tungsten cathodes in a recent EFI experiment [4] produced electron beams with an rms emittance similar to the round-beam emittance required here: (1-2) $\times 10^{-7} \mathrm{~m}-\mathrm{r}$. The current, (1-2) $\mathrm{mA}$, was smaller by an order of magnitude than the start current of $37.2 \mathrm{~mA}$. However, thermionic cathodes producing an order of magnitude higher current density than tungsten are available. For example, $\mathrm{LaB}_{6}$ at $2500-3000 \mathrm{~K}$ produces $20-50 \mathrm{~A} / \mathrm{cm}^{2}$ [25]. Therefore, the flat-beam technique appears to be a promising candidate for producing electron beams satisfying the requirements of a SmithPurcell BWO for terahertz radiation.

Sheet beams have been used previously for millimeter/ submillimeter slow wave generator. For example, Chen et al. [30] have used sheet beam in a Smith-Purcell FEL experiment at $3 \mathrm{~mm}$ wavelength. However, the aspect ratio (horizontal beam size/vertical beam size) in these experiments has been at the most 20 [31] while we require an aspect ratio of 1000 or higher. In addition, the magnitude of the vertical emittance needs to be much smaller. For this reason, it is necessary to employ special techniques such as discussed in this section. We would like to point out that, once the flat beam is produced, one could use the longitudinal magnetic field produced by a solenoid to guide the electron beam, which will be needed to transport the beam maintaining close vicinity to the grating surface.

\section{DISCUSSIONS AND CONCLUSIONS}

In this paper, we have examined the requirements of electron beam parameters for successful operation of a compact terahertz source based on a Smith-Purcell BWO. We have analyzed the three-dimensional mode structure of the surface wave and used it to evaluate the three-dimensional effects correctly. Three-dimensional effects play an important role in determining the start current. The requirements for electron beam parameters were not met in earlier experiments [2,4]. For example, for the Dartmouth experiment [2], the start current from Eq. (19) is $135 \mathrm{~mA}$ if the beam half width $\Delta y$ is taken to be $10 \mu \mathrm{m}$ as reported in Ref. [2]. We also obtain $d I_{s} / d x=$ $39.2 \mathrm{~mA} / \mathrm{mm}$ and $\Delta x=2.2 \mathrm{~mm}$. The requirement for the electron beam's vertical emittance is then $\varepsilon_{y} \leq 7.4 \times$ $10^{-10} \mathrm{~m}$-r. The electron beam current and emittance did not satisfy these criteria in the Dartmouth experiment [2]. Even if these criteria were satisfied, the right-hand side of Eq. (20) for these numbers is 4.3, the inequality is clearly violated, and thus the space-charge effect will significantly deteriorate the electron beam emittance. With the optimized parameters used in Sec. V, the start current is reduced significantly from that required for the
Dartmouth case. The space-charge effect does not therefore deteriorate the electron beam quality significantly.

It is interesting to compare the structure of electromagnetic fields and Poynting vectors in the 2D and 3D analyses presented here. In the $2 \mathrm{D}$ analysis, there will be three components of electromagnetic field $-E_{z}, B_{x}$, and $E_{y}$. The Poynting vector, above the grating surface, will be in the $z$-direction. Inside the grooves, standing waves will be formed. For the 3D case, one can show using Maxwell equations that there will be additional components of electromagnetic fields $-B_{y}$ and $B_{z}$. Hence, the Poynting vector, above the grating surface, will be in the $z$ as well as the $x$-direction. Inside the grooves, standing waves will be formed along the $z$-direction, but there will be a Poynting vector along the $x$-direction. Hence, there will be a flow of energy along the direction of grooves, inside all grooves. Under the paraxial approximation that we have discussed in this paper, however, the components $B_{y}$ and $B_{z}$ will be small and the Poynting vector along $x$-direction will be much smaller compared to that along the $z$-direction. We can therefore primarily assume that flow of power is along the $z$-direction above the grating surface even in the $3 \mathrm{D}$ case.

In this paper we have assumed no reflection at the ends of the grating. When one includes the reflections, the backward wave gets reflected to forward wave at the upstream end and the forward wave gets reflected to the backward wave at the downstream end of the grating. The start current changes due to reflections and, by properly tuning the grating length or the electron beam energy, one can get a reduced start current. An analysis including reflections at the ends of the grating has been performed recently $[32,33]$. In Ref. [32], we have extended our simulation to include reflections. The reflection will be naturally present at the end of the grating, and one can also enhance it with mirrors. For the parameters discussed in this paper, following the analysis presented in Ref. [32], we find that the start current requirement can be relaxed if the reflection is included. If the amplitude reflection coefficient $\mathcal{R}=0.8$, the start current can be reduced from 37.2 to $27.5 \mathrm{~mA}$.

To summarize, we obtained criteria on electron beam properties to operate a Smith-Purcell BWO for terahertz radiation, and an expression for the output power. The derivation is based on the results of a two-dimensional thin beam theory and its three-dimensional extension. Methods to produce electron beams of required characteristics were suggested by means of a line-shaped tungsten cathode or by employing a flat-beam technique on an initially round, high-current thermionic cathode such as $\mathrm{LaB}_{6}$.

\section{ACKNOWLEDGMENTS}

We thank our colleagues for useful discussions; Charlie Brau on the physics of the Smith-Purcell BWO, Albert Crewe and Bud Kapp on electron sources and SEM, and 
Dazhi Li on simulation methods. This work is supported by the U.S. Department of Energy, Office of Science, Office of Basic Energy Sciences, under Contract No. DE-AC0206CH11357.

[1] S. J. Smith and E. M. Purcell, Phys. Rev. 92, 1069 (1953).

[2] J. Urata, M. Goldstein, M. F. Kimmit, A. Naumov, C. Platt, and J. E. Walsh, Phys. Rev. Lett. 80, 516 (1998).

[3] Proceedings of DOE-NSF-NIH Workshop on Opportunities in THz Science, 2004.

[4] O. H. Kapp, Y.-e Sun, K.-J. Kim, and A. V. Crewe, Rev. Sci. Instrum. 75, 4732 (2004).

[5] See, for example, M. Neviere, in Electromagnetic Theory of Gratings, edited by R. Petit (Springer-Verlag, Berlin, 1980).

[6] H. L. Andrews and C.A. Brau, Phys. Rev. ST Accel. Beams 7, 070701 (2004).

[7] See, for example, M. Chodorow and C. Susskind, Fundamentals of Microwave Electronics (McGraw-Hill Inc., New York, 1964).

[8] V. Kumar and K-J. Kim, Phys. Rev. E 73, 026501 (2006).

[9] L. Schachter and A. Ron, Phys. Rev. A 40, 876 (1989).

[10] K. Yasumoto, T. Tanaka, and T. Aramaki, IEEE Trans. Plasma Sci. 18, 699 (1990).

[11] V. Kumar and K-J. Kim, Proceedings of PAC 2005, 2005, p. 1616.

[12] V. Kumar and K.-J. Kim, Proceedings of FEL 2006, 2006, p. 67.

[13] H.L. Andrews, C.H. Boulware, C.A. Brau, and J.D. Jarvis, Phys. Rev. ST Accel. Beams 8, 050703 (2005).

[14] J. A. Swegle, Phys. Fluids 30, 1201 (1987).

[15] J. T. Donahue and J. Gardelle, Phys. Rev. ST Accel. Beams 8, 060702 (2005).

[16] D. Li and K. Imasaki, Proceedings of FEL 2006, p. 434.
[17] D. Li, Z. Yang, K. Imasaki, and G.-S. Park, Phys. Rev. ST Accel. Beams 9, 040701 (2006).

[18] J. T. Donohue and J. Gardelle, Phys. Rev. ST Accel. Beams 9, 060701 (2006).

[19] D. Li, K. Imasaki, Z. Yang, and G.-S. Park, Appl. Phys. Lett. 88, 201501 (2006).

[20] V. Kumar and K.-J. Kim (to be published).

[21] See K.-J. Kim, Characteristics of Synchrotron Radiation, AIP Conference Proceedings No. 184 (AIP, New York, 1989), p. 565.

[22] A. Chao, Physics of Collective Beam Instabilities in High Energy Accelerators (John Wiley \& Sons, Inc., New York, 1993).

[23] H.L. Andrews, C.H. Boulware, C. A. Brau, and J. D. Jarvis, Phys. Rev. ST Accel. Beams 8, 110702 (2005).

[24] V. Kumar, K.-J. Kim, and D. Li, APAC2007.

[25] L. Reimer, Transmision Electron Microscopy, Springer Series in Optical Sciences (Springer Verlag, Berlin, 1997), Vol. 36.

[26] R. Brinkmann, Y. Derbenev, and K. Flöttmann, Phys. Rev. ST Accel. Beams 4, 053501 (2001).

[27] K.-J. Kim, Phys. Rev. ST Accel. Beams 6, 104002 (2003).

[28] D. Edwards, H. Edwards, N. Holtkamp, S. Nagaitsev, J. Santucci, R. Brinkmann, K. Desler, K. Flöttmann, I. Bohnet, and M. Ferrario, Proceedings of LINAC 2000, p. 122.

[29] P. Piot, Y.-E. Sun, and K.-J. Kim, Phys. Rev. ST Accel. Beams 9, 031001 (2006).

[30] J. Chen, L. Zheng, Y. Zhang, and Z. Yang, Int. J. Electron. 88, 467 (2001).

[31] B. Carlsten (private communication).

[32] V. Kumar and K.-J. Kim, Proceedings of FEL 2006, p. 71.

[33] H. L. Andrews, C. H. Boulware, C. A. Brau, J. T. Donohue, J. Gardelle, and J. D. Darvis, New J. Phys. 8, 289 (2006). 